\title{
Very rare giant coronary aneurysm
}

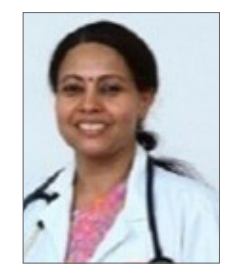

Rajeshwari Nayak*, DNB; Yeramma Vijayachandra Reddy, MD, DM, MRCP; Girinath Maligail Ramakrishnan, MS, Mch, FRCS; Rochita Ramanan, MD; Sathyamurthy Immineni, MD, DM; Jenifer Johnson, BS

Apollo Hospitals, Chennai, India

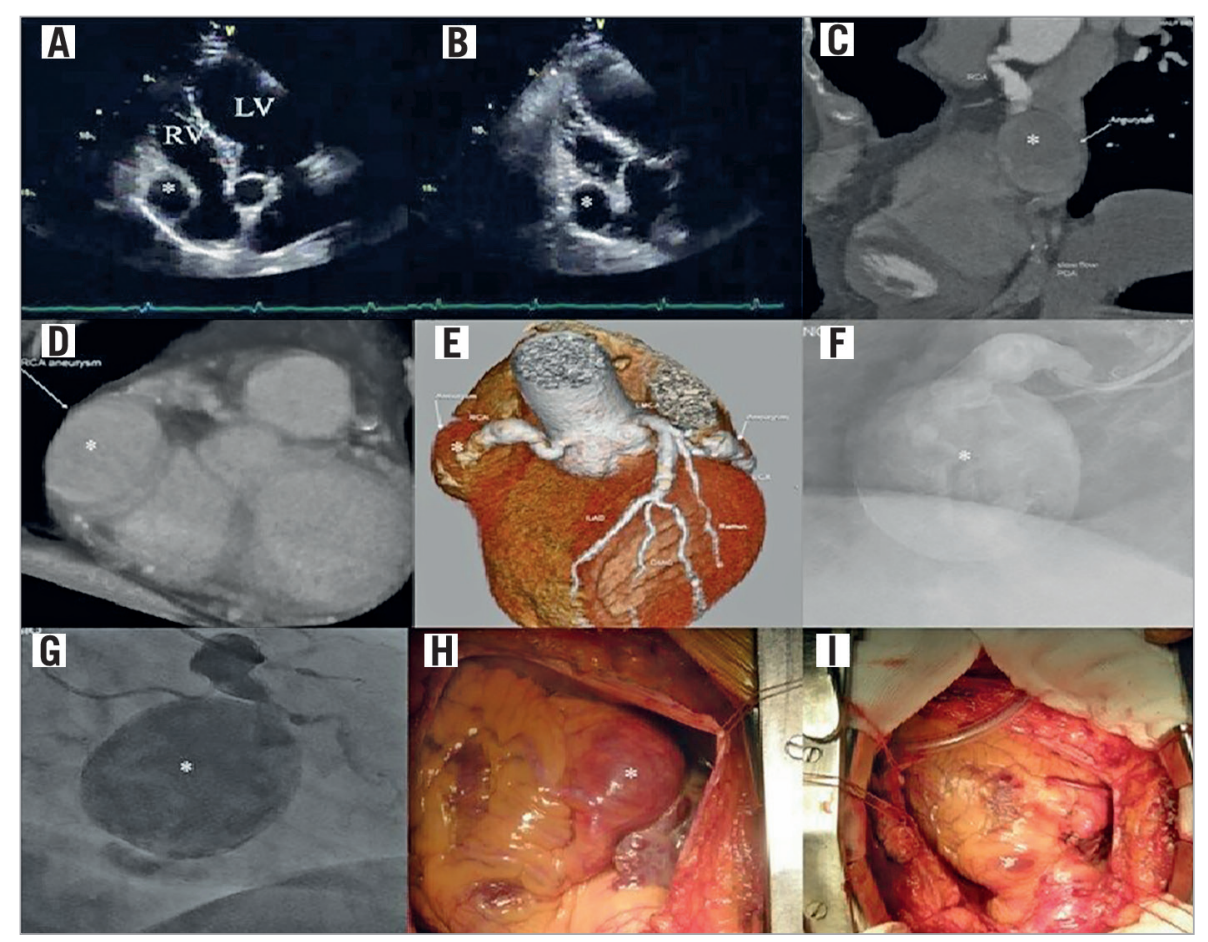

*Corresponding author: Apollo Hospitals, No.21, Greams Lane, off. Greams Road, Chennai, Tamilnadu, India. E-mail: drrajeshwari2006@yahoo.com 
Coronary artery aneurysms (CAA) are rare and giant CAA are even rarer. We describe an asymptomatic patient with a giant CAA of the right coronary artery (RCA). A 65-year-old underwent echography (Panel A \& Panel B), as part of his medical evaluation for a life insurance policy, which revealed a lesion in the right atrium (RA).

A coronary angiogram revealed a giant aneurysm of the midsegment of the RCA (Panel F \& Panel G). CT coronary angiogram (CTA) revealed a large fusiform aneurysm, measuring $32.1 \mathrm{~mm} \times 46 \mathrm{~mm}$ (Panel C, Panel D \& Panel E).

Management options of percutaneous coronary intervention (PCI) with covered stent and surgical repair were discussed. PCI was not a viable option as the artery proximal and distal to the aneurysm appeared highly ectatic.

He underwent surgical repair (Panels H\&l). A coronary artery bypass graft was performed using saphenous vein grafts to the posterior descending artery and the acute marginal branches.
The RCA proximal and distal to the aneurysm was ligated. A third ligature was placed around the origin of the acute marginal branch, achieving complete isolation of the aneurysm.

At 1-year follow-up, echography showed a persisting RCA aneurysm. CTA showed an aneurysm measuring $40 \mathrm{~mm} \times 32 \mathrm{~mm}$, partially thrombosed, with evidence of persistent filling from the native RCA. The large size of the aneurysm and the persistent filling by the RCA placed the patient at considerable risk of aneurysmal rupture and sudden cardiac death.

The patient underwent repeat surgery and the RCA was ligated totally at its origin. At 1-year follow-up after the second surgery, there was no echocardiographic evidence of a persisting lesion. CTA revealed a fully thrombosed RCA aneurysm.

\section{Conflict of interest statement}

The authors have no conflicts of interest to declare. 